\title{
Potential-well distortion, microwave instability, and their effects with colliding beams at KEKB
}

\author{
Yunhai Cai \\ SLAC National Accelerator Laboratory, Stanford University, Menlo Park, California 94025, USA \\ J. Flanagan, H. Fukuma, Y. Funakoshi, T. Ieiri, K. Ohmi, K. Oide, and Y. Suetsugu \\ KEK, Oho, Tsukuba, Ibaraki 305-0801, Japan \\ Jamal Rorie \\ University of Hawaii at Manoa, Honolulu, Hawaii 96822, USA
}

(Received 25 February 2009; published 30 June 2009)

\begin{abstract}
Microwave instability in the low energy ring of KEKB was studied using a broadband impedance model. The model gave excellent descriptions of longitudinal dynamics for both positive and negative momentum compactions. Moreover, it predicted that the threshold of microwave instability was a factor of 2 lower than the machine nominal operating bunch current. The prediction was confirmed by a measurement using the Belle detector. Furthermore, we integrated the longitudinal wakefield into the beam-beam simulation and applied it to study the combined effects in KEKB. As a result, the beam-beam simulation became truly three dimensional with emittance growth in all three dimensions simultaneously as the beam currents increase. In addition, an observed mystery of asymmetry in the horizontal scan could also be explained by our simulations.
\end{abstract}

DOI: 10.1103/PhysRevSTAB.12.061002

PACS numbers: 71.45. $-\mathrm{d}, 29.27 .-\mathrm{a}, 52.59 .-\mathrm{f}$

\section{INTRODUCTION}

Collisions with a horizontal crossing angle have become an important feature in the design of modern colliders [14], since the success of KEKB [5] in achieving the record luminosity of $1.7 \times 10^{34} \mathrm{~cm}^{-2} \mathrm{~s}^{-1}$ in $e^{+} e^{-}$storage rings. After many years of technological development, two superconducting crab cavities were successfully installed two years ago into two storage rings in KEKB to double its luminosity [6]. Although the crab crossing improves the specific luminosity at low beam currents, the luminosity so far remains below the record of peak luminosity achieved without the crab cavities. It is extremely important for us to understand why. This provided the initial motivation for our studies.

In this paper, we would like to have an independent assessment of possible machine performance and to validate or invalidate the previous results of simulation. Given the duration of the time of our study, we limited ourselves to studying an idealized collider. As a consequence, our result should be considered as an upper limit for the machine performance. Given the nature of this study, we used different methods where possible. For example, rather than using the Lorentz boost to treat the crossing angle, we used a Lie transformation with an intuitively geometrical interpretation.

There are several motivations to include the nontrivial longitudinal beam dynamics into the beam-beam simulation. First, it is well known that an electron beam lengthens as its intensity increases in the presence of a longitudinal wakefield either due to the potential-well distortion or the microwave instability. Second, the bunch length affects several important beam-beam effects including the hourglass effect, phase averaging, and the Piwinski angle. At present, we use a Gaussian with a measured bunch length to approximate these effects in the simulation. It is clear that, in general, the longitudinal bunch shape is not a Gaussian, especially at high intensity, and therefore one has to include the longitudinal wake in the simulation. A particular motivation for us is that the asymmetry in the longitudinal distributions may lead to the other asymmetries observed [7] at KEKB.

In Sec. II, we will start with an introduction to the longitudinal beam dynamics including the wakefield and continue with a study of the potential-well distortion using the Haissinski integral equation. To understand the microwave instability, we will briefly introduce the particle-incell (PIC) method for simulation and apply the simulations to construct an impedance model for the storage rings at KEKB. The results of simulations will be compared to various measurements. At the end of Sec. II, we will introduce a new method to measure the energy spreads for the colliding beam and compare the measurement to a prediction of the simulation.

In the second part of this paper, we will introduce several new features in our beam-beam simulation including a different treatment of crossing angle and crab cavity. Finally, we will show the results of our simulations and their comparisons to the measurements.

\section{LONGITUDINAL BEAM DYNAMICS}

Let us consider an electron in a storage ring executing a small synchrotron oscillation in a stationary rf bucket. For 
simplicity, we introduce a normalized coordinate system, $q=z / \sigma_{z}$ and $p=-\delta / \sigma_{\delta}$, where $z$ is the differential position relative to the synchronized particle with energy $E_{0}, \delta=\left(E-E_{0}\right) / E_{0}$, and $\sigma_{z}$ and $\sigma_{\delta}$ are the standard deviations of position and relative energy in the equilibrium Gaussian distribution at zero beam current. Here we use positive $q$ as the forward direction of the beam. It is well known [8] that the bunch length $\sigma_{z}=\alpha c \sigma_{\delta} / \omega_{s}$, where $\omega_{s}$ is the angular frequency of the synchrotron oscillation and $\alpha$ is the momentum compaction factor. The motion of the electron is that of a simple harmonic oscillator described by the Hamiltonian, $H=\frac{1}{2}\left(q^{2}+p^{2}\right)$, along with independent variable $\theta=\omega_{s} t$.

In general, the electron also experiences a collective force induced by the bunch distribution $\lambda(q)$. Using the notion of an integrated wakefield $W(q)$ [9] in a single turn, the dynamics can be described by a Hamiltonian

$$
H=\frac{1}{2}\left(q^{2}+p^{2}\right)-I_{n} \int_{-\infty}^{q} d q^{\prime \prime} \int_{-\infty}^{\infty} d q^{\prime} \lambda\left(q^{\prime}\right) W\left(q^{\prime \prime}-q^{\prime}\right),
$$

where

$$
I_{n}=\frac{r_{e} N_{b}}{2 \pi \nu_{s} \gamma \sigma_{\delta}}
$$

is the normalized current, which was introduced by Oide and Yokoya [10]. $N_{b}$ represents the number of electrons in the bunch, $\nu_{s}$ is the synchrotron tune, $r_{e}$ is the classic radius of electron, and $\gamma=E_{0} / m c^{2}$. Here, the bunch distribution $\lambda(q)$ has been normalized, namely $\int_{-\infty}^{\infty} \lambda(q) d q=1$.

It is worth noting that the dynamics effect of the wakefield is scaled by the normalized current $I_{n}$. Its dependence on the parameters in Eq. (2) clearly shows that we prefer a higher energy, faster synchrotron oscillation, or larger relative energy spread to reduce the effects of the wakefield. Although, it does not explicitly depend on the momentum compaction factor $\alpha$, for a negative $\alpha<0$, one needs to use a negative normalized current as well, namely $I_{n}<0$. Moreover, if $W(q)$ is given in terms of $\mathrm{V} / \mathrm{pC}$, one should convert $I_{n}$ from meter to $\mathrm{pC} / \mathrm{V}$.

Furthermore, it can be shown that the evolution of beam density distribution $\Psi(q, p)$ is governed by the VlasovFokker-Planck (VFP) equation

$$
\frac{\partial \Psi}{\partial \theta}-\{H, \Psi\}_{\mathrm{PB}}=2 \beta \frac{\partial}{\partial p}\left(p \Psi+\frac{\partial \Psi}{\partial p}\right),
$$

where $\beta=1 / \omega_{s} \tau_{d}$ and $\tau_{d}$ is the longitudinal damping time. We use the subscript PB to indicate the Poisson bracket. Actually, $H$ is the Hamiltonian defined in Eq. (1) with the substitution of $\lambda(q)=\int_{-\infty}^{\infty} \Psi(q, p) d p$. As a result, the VFP equation is a nonlinear integral and partial differential equation. In general, it can only be solved by numerical methods [11]. In fact, it is a special form of the Fokker-Planck equation since the damping and diffusion terms on the right-hand side involve only the partial derivatives of $p$. This is a consequence of the fact that the synchrotron radiation causes loss and quantum diffusion only in the energy of the radiating electron not in its time of flight.

\section{A. Haissinski distributions}

Historically, it was Haissinski who discovered that the VFP equation [Eq. (3)] has a static solution in the form of [12]

$$
\Psi_{0}(q, p)=\frac{1}{\kappa \sqrt{2 \pi}} \exp \left(-H_{0}\right)=\lambda_{0}(q) \exp \left(-\frac{p^{2}}{2}\right) / \sqrt{2 \pi} \text {. }
$$

Here the subscript " 0 " indicates that the solution does not explicitly depend on $\theta$ or $\partial \Psi / \partial \theta=0$. Since $\Psi_{0}$ is a function of the Hamiltonian $H_{0}$ only, it commutes with $H_{0}$ in the Poisson bracket; therefore the right-hand side of the equation vanishes by itself.

On the other hand, $\Psi_{0}$ is also factorized into a product of a Gaussian distribution in $p$ and $\lambda_{0}(q)$, which makes the right-hand side of the equation vanish separately. Using Eq. (1) for $H_{0}$ and eliminating the dependence of $p$ in Eq. (4), we find the well-known Haissinski integral equation,

$$
\begin{aligned}
\lambda_{0}(q)= & \exp \left[-\frac{q^{2}}{2}+I_{n} \int_{-\infty}^{q} d q^{\prime \prime} \int_{-\infty}^{\infty} d q^{\prime} \lambda_{0}\left(q^{\prime}\right)\right. \\
& \left.\times W\left(q^{\prime \prime}-q^{\prime}\right)\right] / \kappa,
\end{aligned}
$$

where $\kappa$ is a constant determined by the normalization condition, $\int_{-\infty}^{\infty} \lambda_{0}(q) d q=1$. At zero current, $I_{n}=0$, so the solution becomes a Gaussian. In general, this nonlinear integral equation can be solved numerically using Newton's iteration starting from the Gaussian distribution. Haissinski solutions, due to the wakefield of a broadband resonance model $\left(Q=1, x_{r}=\omega_{r} \sigma_{z} / c=3\right.$, wake amplitude $w_{0}=5 \times 10^{5} \mathrm{~m}^{-1}$ ), are shown in Fig. 1 .

In practice, we know that the Haissinski distribution is not just a possible solution but also the equilibrium distribution of the VFP equation at a sufficiently low current. Above a threshold of $I_{n}$, the Haissinski distribution is no longer a stable solution. In the literature, the associated instability is commonly referred to as the microwave instability.

\section{B. Simulation with macroparticles}

In general, we have to solve the VFP equation numerically. The equation can be solved [11] using a twodimensional grid to represent the distribution in phase space. Since we need to deal with distributions in sixdimensional phase space in the beam-beam simulation, we have to introduce macroparticles to represent the phase-space distribution, 


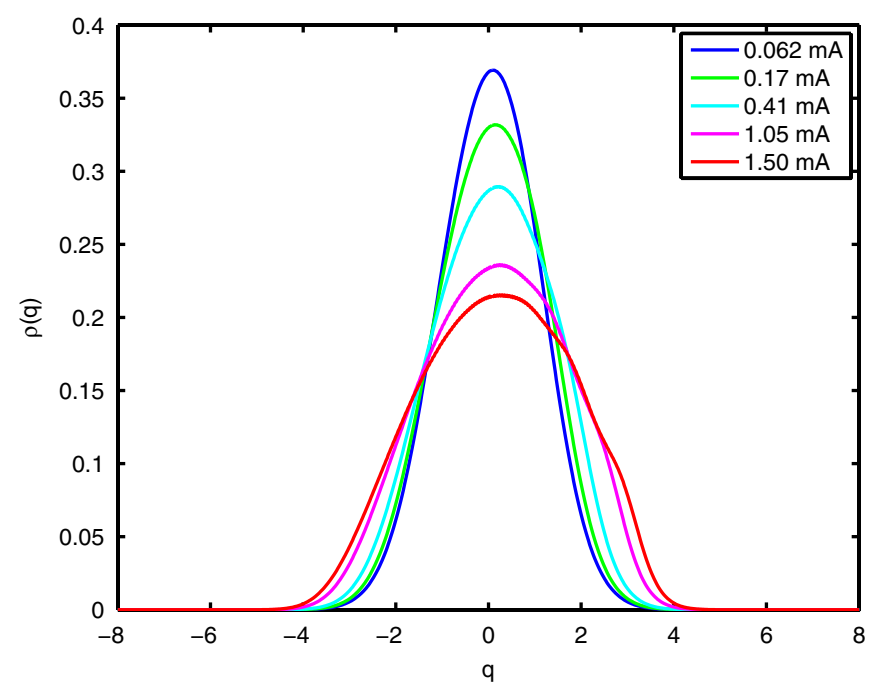

FIG. 1. (Color) Haissinski distributions at various positron bunch currents in the low energy ring of KEKB.

$$
\Psi(q, p)=\frac{1}{N_{p}} \sum_{i=1}^{N_{p}} \delta\left(q-q_{i}\right) \delta\left(p-p_{i}\right),
$$

where $q_{i}, p_{i}$ are the canonical coordinates of the particles so that the evolution of $\Psi(q, p)$ can be carried out by tracking the particles.

For each step of $\Delta \theta$, we use three integration steps based on the technique of splitting operators. First, we apply a kick generated by the wakefield $W(q)$ :

$$
\Delta p_{i}=I_{n} \int_{-\infty}^{\infty} d q^{\prime} \lambda\left(q^{\prime}\right) W\left(q_{i}-q^{\prime}\right) \Delta \theta .
$$

To speed up the calculation, we deposit the charge of every particle onto two adjacent grid points with a linear weighting to accumulate $\lambda(q)$ on a one-dimensional mesh. The integration is replaced by a summation over the grid and the kicks on all the grid points are calculated and stored. For the kick on the particle, we use a linear interpolation of kicks on the two adjacent grids. In the second step, we simply have a rotation

$$
\begin{aligned}
& q_{i}=\cos (\Delta \theta) q_{i}+\sin (\Delta \theta) p_{i}, \\
& p_{i}=-\sin (\Delta \theta) q_{i}+\cos (\Delta \theta) p_{i}
\end{aligned}
$$

which is a solution of the free harmonic oscillator. In the final step, we apply the radiation damping and quantum excitation [13]

$$
\Delta p_{i}=-2 \beta p_{i} \Delta \theta+\sqrt{12 \beta \Delta \theta} \xi(i)
$$

where $\xi$ is a random number generated by a uniform distribution between -1 to 1 . As an example shown in Fig. 2, we make a direct comparison between the VFP solver [11] and our PIC simulation. One can see from the figure that there is not much difference and both give a

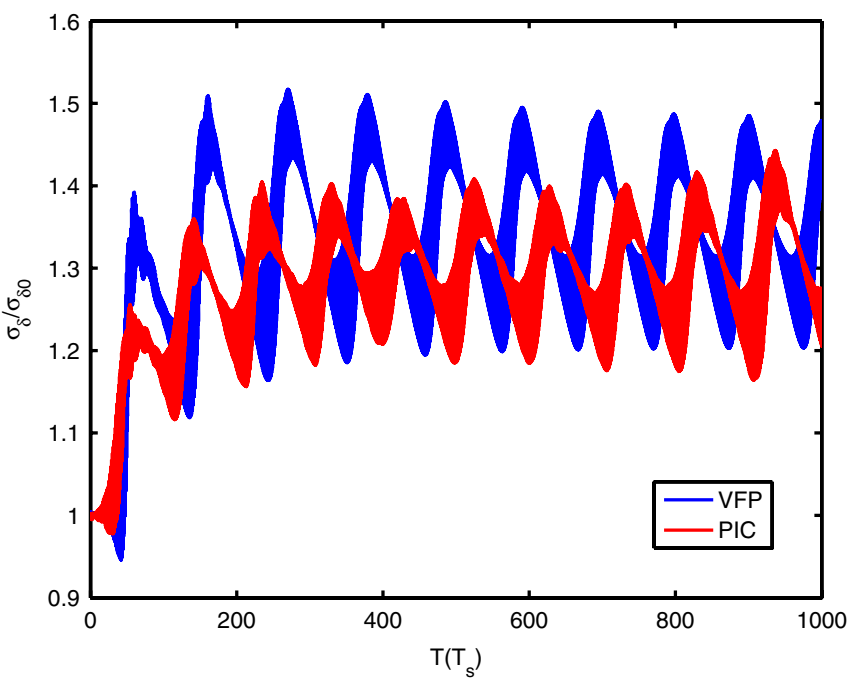

FIG. 2. (Color) A comparison between VFP and PIC codes using the impedance of SLC damping ring [21]. The number of macroparticles is chosen to be the same as the number of grid points in the VFP solver.

good description of the saw-tooth instability including its periodicity.

\section{Impedance models}

In general, one needs to collect all possible impedance sources, such as bellows, masks, and collimators, and calculate their impedance, and construct a wakefield $W(q)$ by adding up all contributions in the entire storage ring. Clearly, this task is very demanding, could take a long time to complete, and often some impedance is left uncounted. Nevertheless, a detailed impedance model is being built but is yet to be completed.

For the purpose of the beam-beam simulations, we are not so interested in the detail of impedance sources as much as the effective longitudinal distributions so that they can be correctly modeled. Therefore, we will take an alternative and construct a broadband impedance model from the measured beam profiles at various beam currents. For simplicity, we choose the $Q=1$ broadband resonance model.

For a broadband impedance model with a parallel $L R C$ circuit, the nonvanishing wakefield, for $q<0$, is given by [9]

$$
W(q)=w_{0}\left[\cos (A q)+\sin (A q) / \sqrt{4 Q^{2}-1}\right] \exp \left(x_{r} q / 2 Q\right)
$$

where $A=x_{r} \sqrt{1-1 / 4 Q^{2}}$ and $x_{r}=\omega_{r} \sigma_{z} / c$. One can easily convert three dynamical parameters $Q, x_{r}, w_{0}$ to their engineering counterparts $L, R, C$ by using

$$
L=w_{0}\left(\sigma_{z} / x_{r} c\right)^{2},
$$



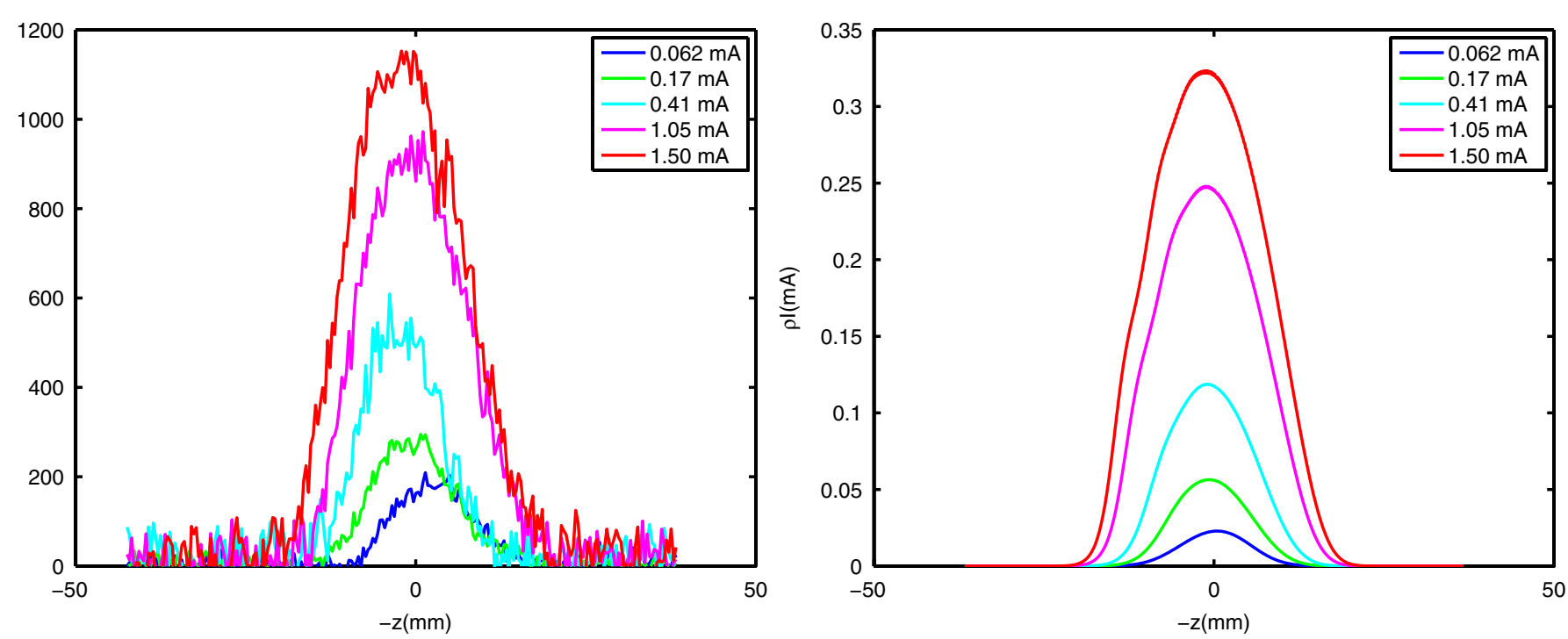

FIG. 3. (Color) Comparisons of bunch profiles of the measurement using a streak camera and products of the Haissinski distributions and the corresponding beam currents.

$$
\begin{gathered}
R=Q w_{0}\left(\sigma_{z} / x_{r} c\right), \\
C=1 / w_{0} .
\end{gathered}
$$

It has been known from previous work by Ieiri and Koiso [14] that both rings, like many modern storage rings, were rather inductive. By fitting to a pure inductance impedance model, they found that $L=96 \mathrm{nH}$ in the LER and $L=$ $104 \mathrm{nH}$ in the HER, respectively. These inductances more or less fix another parameter in the broadband model. However, there is still a tradeoff between $x_{r}$ and $w_{0}$ to be made according to Eq. (11). The necessary information is provided by the measurement of the positron beam profiles using a streak camera. The data is shown in the plot on the left of Fig. 3. It is clear that the measured shapes are essentially Gaussians; we have to choose $x_{r} \geq 2$ to avoid a shoulder in the distributions at high currents. In the plot on the right of Fig. 3, we show the Haissinski distributions times the beam currents at the corresponding currents.

The final selection of the parameters $x_{r}$ and $w_{0}$ are actually made using a compromise between the fitting to the measured bunch lengths shown in Fig. 4 and the matching to the beam profiles. We settle on the values of $x_{r}=3$ and $w_{0}=5 \times 10^{5} \mathrm{~m}^{-1}$. The results of the fitting to the measurements are shown in the plot on the left of Fig. 4. Note that the PIC simulations are necessary to fit the measured bunch lengths.
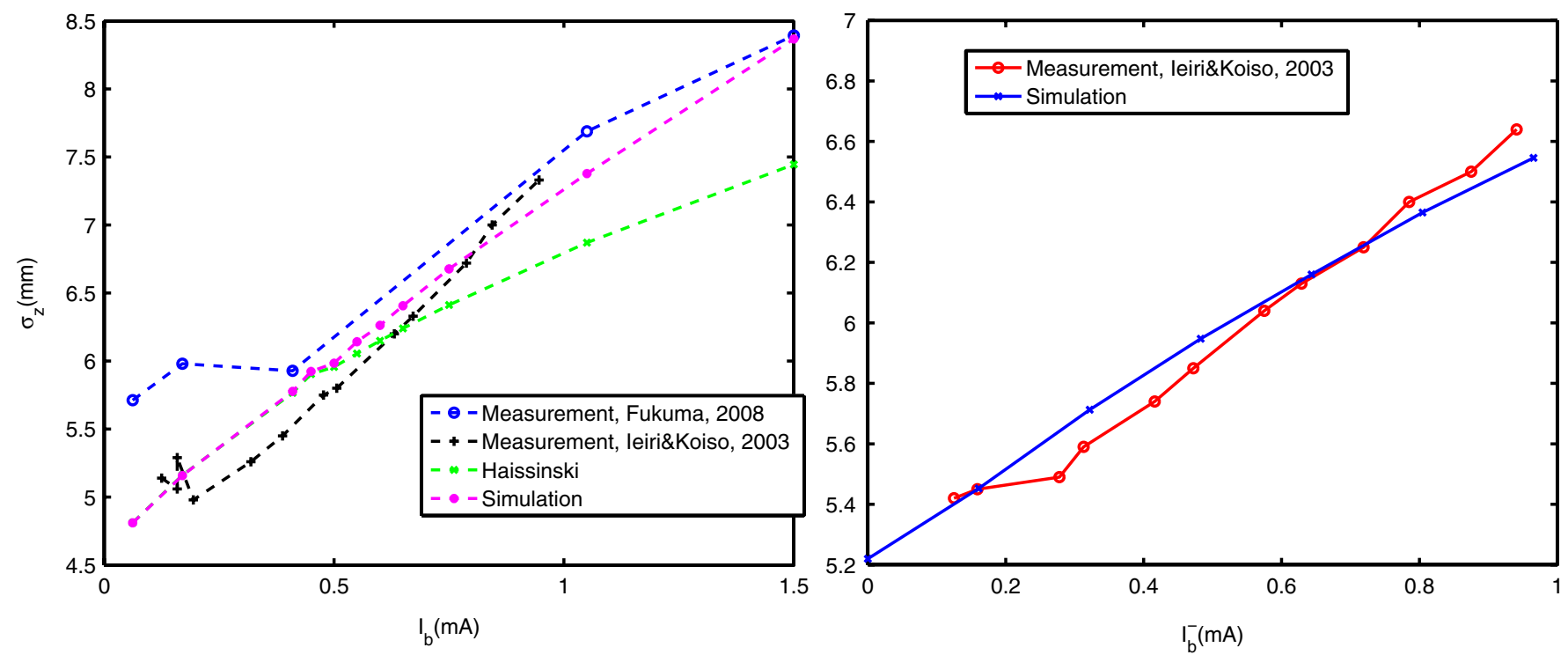

FIG. 4. (Color) Comparisons of bunch length from measurement, calculation, and simulation. The figure on the left is for the LER and the right for the HER. 


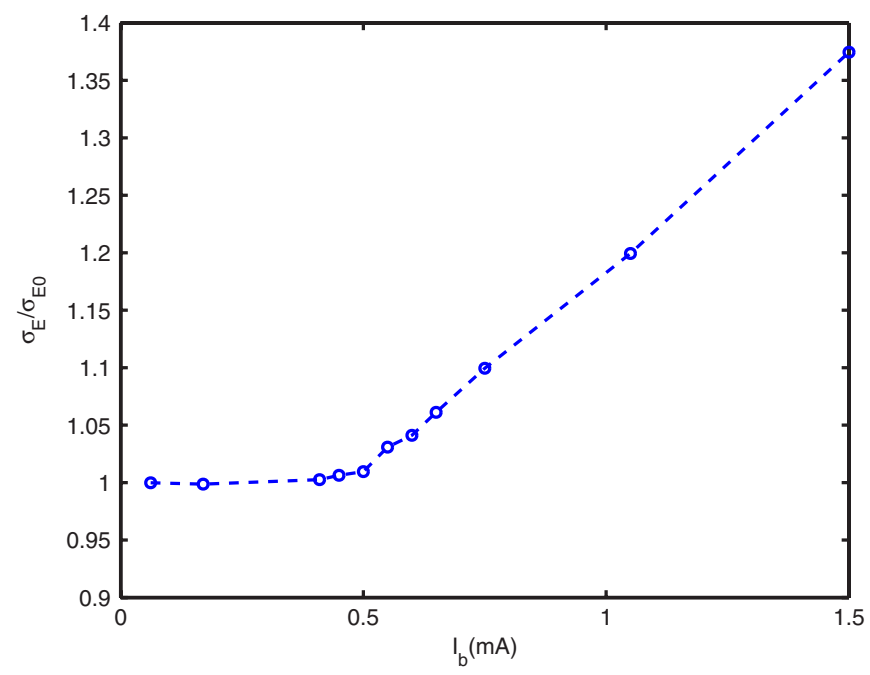

FIG. 5. (Color) Normalized energy spread as a function of the bunch current in the LER of KEKB. It shows that the threshold of microwave instability is about $0.5 \mathrm{~mA}$.

As one can see from the plot for the LER, the simulated distributions start to deviate from the Haissinski distributions beyond the value $0.5 \mathrm{~mA}$ of the bunch current. According to the theory of microwave instability, the threshold is $I_{\text {th }}=0.5 \mathrm{~mA}$. At the operating current of $1.0 \mathrm{~mA}$, we have a $20 \%$ increase of energy spread in the simulation as shown in Fig. 5. However, we are puzzled by the fact that there is no obvious turning of the bunch length at the threshold. This puzzle is one of the exceptions and may be explained by the particular impedance we used in the simulation. Because of this kind of exception, we defined the threshold using the change of the energy spread and tried to validate the threshold by the measurement of the energy spread. Also, as one can see in Fig. 4, the bunch lengths obtained by simulations and solving the Haissinski integral equation start to deviate above the threshold.

One pleasant surprise is that the impedance model also gives a good agreement to the measurement when $\alpha$ switches to be negative. Furthermore, the simulation with negative $\alpha$ shows an unstable and periodically bursting mode above $0.6 \mathrm{~mA}$, which is consistent with the observation. The threshold is nearly doubled if we double the value of negative $\alpha=-6.66 \times 10^{-4}$.

For the HER, there are no measurements of bunch shapes so we chose $x_{r}=2$ and $w_{0}=1.6 \times 10^{5} \mathrm{~m}^{-1}$. The result of the fitting is shown in the plot on the right of Fig. 4. The parameters of the impedance models are tabulated in Table I. The values of the inductances in the table are slightly higher than those given by Ieiri and Koiso. They are consistent with the calculated inductance [15] in PEP-II if we scale them with the ratio of their circumferences. However, they are nearly a factor of 4 larger than those given in the KEKB design report [5]. The discrepancy indicates that there are missing sources of
TABLE I. Parameters of the $L R C$ impedance model for both rings.

\begin{tabular}{llcc}
\hline \hline Parameter & Description & LER & HER \\
\hline$L(\mathrm{nH})$ & Inductance & 116.7 & 109.1 \\
$R(\mathrm{~K} \Omega)$ & Resistance & 22.9 & 12.5 \\
$C(\mathrm{fF})$ & Capacitor & 0.22 & 0.69 \\
\hline \hline
\end{tabular}

impedance or the coherent synchrotron radiation may play a role in the longitudinal dynamics when $\sigma_{z}=5 \mathrm{~mm}$.

\section{D. $\mathbf{Y}(2 S)$ and energy spreads}

It is well known that there are many narrow $\Upsilon(n S)$ resonances in the $B \bar{B}$ system. In particular, $\mathrm{Y}(2 S)$ has a mass of $m_{2 s}=10.02330 \pm 0.00031 \mathrm{GeV}$ and a full width of $\Gamma=44 \pm 7 \mathrm{keV}$, which is much less than the nominal energy spreads of $\sigma_{E}^{+}=2.54 \mathrm{MeV}$ and $\sigma_{E}^{-}=5.34 \mathrm{MeV}$ in the positron and electron beams, respectively. This narrow resonance allows us to extract the change of the energy spread in the beams.

In a high energy $e^{+} e^{-}$collider such as KEKB, the total energy $E_{\text {c.m. }}$ in the center-of-mass and the available energy is given by $E_{\text {c.m. }}=\sqrt{4 E^{+} E^{-}}$, where $E^{+}$and $E^{-}$are the energy of the colliding positron and electron, respectively. To generate the $\Upsilon(2 S)$, one needs to set the energies $E_{0}^{+}$ and $E_{0}^{-}$of the rings such that

$$
m_{2 s} c^{2}=\sqrt{4 E_{0}^{+} E_{0}^{-}}
$$

for the on-momentum particles. Furthermore, for any pair of off-momentum particles with their energy off by $\Delta E^{+}$ and $\Delta E^{-}$, they should satisfy the condition

$$
m_{2 s} c^{2}=\sqrt{4\left(E_{0}^{+}+\Delta E^{+}\right)\left(E_{0}^{-}+\Delta E^{-}\right)},
$$

here we have ignored the width of the resonance. Dividing Eq. (14) from Eq. (15) and then squaring, we obtain

$$
\left(\delta^{+}+1\right)\left(\delta^{-}+1\right)=1,
$$

where $\delta^{ \pm}=\Delta E^{ \pm} / E_{0}^{ \pm}$. Since $\delta^{ \pm} \ll 1$, Eq. (16) essentially reduces to $\delta^{+}+\delta^{-}=0$. Now, let us consider two colliding beams with Gaussian energy distributions

$$
\rho\left(\delta^{ \pm}\right)=e^{-\left(\delta^{ \pm}\right)^{2} / 2\left(\sigma_{\delta}^{ \pm}\right)^{2}} / \sqrt{2 \pi} \sigma_{\delta}^{ \pm},
$$

and calculate a probability of all colliding pairs that can generate the $\Upsilon(2 S)$. One easily sees that the probability is proportional to a double integral

$$
\begin{gathered}
\int_{-\infty}^{\infty} d \delta^{+} \int_{-\infty}^{\infty} d \delta^{-} \rho\left(\delta^{+}\right) \rho\left(\delta^{-}\right) \delta\left(\delta^{+}+\delta^{-}\right) \\
=\frac{1}{\sqrt{2 \pi} \sqrt{\left(\sigma_{\delta}^{-}\right)^{2}+\left(\sigma_{\delta}^{+}\right)^{2}}} .
\end{gathered}
$$

It is clear that smaller energy spreads of the colliding 


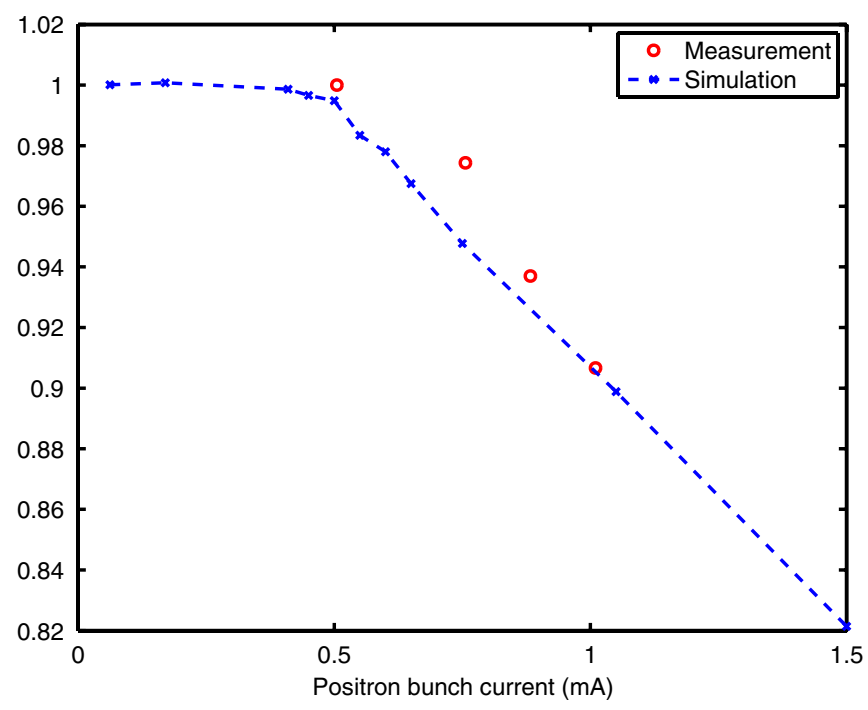

FIG. 6. (Color) Ratio of hadron events to Bhabha was measured as a function of positron bunch current using the Belle detector. The data is normalized to the measured value at $0.5 \mathrm{~mA}$. The result of simulation is plotted according to Eq. (18) and the energy spreads at zero currents are used for the normalization.

beams generate more events on the extremely narrow resonance. This result allows us to measure the energy spread of the beam from the hadron events that are uniquely associated with the $Y(2 S)$ resonance. Fortunately, KEKB was operated at this resonance for its physics run and therefore provided us an excellent opportunity to measure the change of the energy spread as a function of the beam current in the LER using the Belle detector.

We started the positron beam current at half of its nominal operating value for the measurement. The corresponding bunch current was $0.5 \mathrm{~mA}$, which is the predicted threshold of the microwave instability. Further reduction of the current was possible but with a significant reduction of the luminosity. The electron beam current was kept at its nominal value throughout the experiment. The result of the measurement and the comparison to the simulation are shown in Fig. 6. We have excellent agreement. Since the luminosity changed as we changed the beam current, it is necessary to take the ratio to the number of the Bhabha events, which has a very weak dependence on the energies and is proportional to the luminosity. In the simulation, we used the impedance models tabulated in Table I. In order to make the comparison to the measurement, we also used the energy spreads at zero beam currents listed in Table III.

\section{TREATMENT OF A CROSSING ANGLE}

A common treatment of crossing angle is to use the "Lorentz boost" introduced by Hirata [16] in the context of the strong-weak approximation and later by Ohmi [17] in the strong-strong simulation. Here we will introduce a different method based on Lie operators.

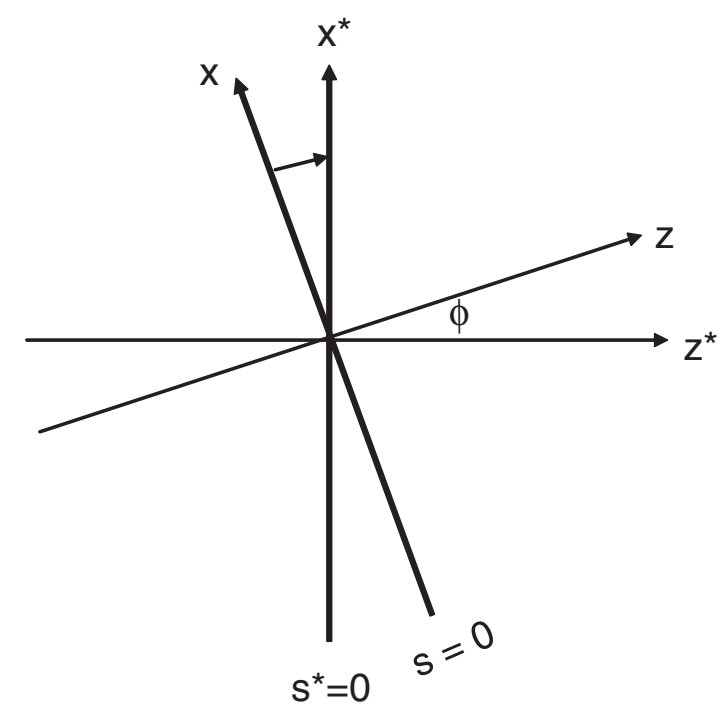

FIG. 7. A rotation around the $y$ axis presented by a Lie operator.

\section{A. Rotation around $\boldsymbol{y}$ axis}

Let us use $x, p_{x}, y, p_{y}, \delta$, and $l$ to denote the canonical coordinates of a charged particle, where $x, y$ are the transverse displacements, $\delta$ is the relative momentum deviation, and $l$ is the time of flight (in unit of length) relative to the synchronous particle. To handle colliding beams with a horizontal crossing angle, we need a transformation that rotates the particles in a single slice $(s=0)$ to the head-on frame $\left(s^{*}=0\right)$ as illustrated in Fig. 7. It is clear that the axis of the rotation is the $y$ axis. It is well known [18] that this transformation can be generated by the Lie operator: $\quad \mathcal{R}_{y}(\phi)=\exp \left(: x p_{s}: \phi\right)$, where $p_{s}=$ $\sqrt{(1+\delta)^{2}-p_{x}^{2}-p_{y}^{2}}$.

The explicit transformation can be obtained by solving the Hamiltonian's equations with $H=-x p_{s}$ and $\phi$ as the independent variable. It can be written as follows:

$$
\begin{aligned}
x^{*} & =\frac{x p_{s}}{\cos \phi\left(p_{s}-p_{x} \tan \phi\right)}, \\
p_{x}^{*} & =p_{x} \cos \phi+p_{s} \sin \phi, \\
y^{*} & =y+\frac{x p_{y} \tan \phi}{\left(p_{s}-p_{x} \tan \phi\right)}, \\
p_{y}^{*} & =p_{y}, \quad \delta^{*}=\delta, \\
l^{*} & =l+\frac{x(1+\delta) \tan \phi}{\left(p_{s}-p_{x} \tan \phi\right)} .
\end{aligned}
$$

Since it is the exact solution of the Hamiltonian's equation, it is symplectic. In addition to the rotation operator, we need the horizontal shift operator $\mathcal{S}_{x}(\delta x)=$ $\exp \left(: p_{x}: \delta x\right)$ and the drift operator $\mathcal{D}_{z}(\delta s)=\exp \left(: p_{s}: \delta s\right)$. 


\section{B. Colliding process}

For every collision, the macroparticles are assigned to the slices according to their longitudinal positions. Since the beam distributions are dynamically evolved during the collision, the sequence of the colliding slices is identical to the time sequence.

For a given pair of colliding slices at $z^{ \pm}=-l^{ \pm}$, we need to compute where the collision actually occurs: $s^{ \pm}=$ $\left(z^{ \pm}-z^{\mp}\right) / 2$. Since the reference point for all particles is the interaction point, we first need to transport the particles in the slices to the actual collision point by the drift operator $\mathcal{D}_{z}^{ \pm}\left(s^{ \pm}\right)=\mathcal{D}_{z}\left(s^{ \pm}\right)$so that the hourglass and phase-average effects due to a finite bunch length are properly included in the simulation.

As illustrated in Fig. 8, in order to transport the particles to the coordinate system where the beam-beam force is computed, we need to perform three additional transformations. First, we make a rotation $\mathcal{R}_{y}^{ \pm}(\phi)=\mathcal{R}_{y}( \pm \phi)$ to an upright coordinate and then follow up with a transformation to make a horizontal shift to the side of the opposing beam using the shift operator $\mathcal{S}_{x}^{ \pm}\left(s^{ \pm}, \phi\right)=$ $\mathcal{S}_{x}\left(-2 s^{ \pm} \sin \phi\right)$. Finally, we make another rotation $\mathcal{R}_{y}^{ \pm}(\phi)$.

Using the particle distributions of the opposing beam at the collision point, we compute the beam-beam force by solving the two-dimensional Poisson equation [19]. The integrated beam-beam kick is applied as

$$
\begin{aligned}
& \Delta p_{x}^{ \pm}=-\frac{e}{E_{0}^{ \pm}} \int_{\text {slice }} E_{x}^{\mp} d s, \\
& \Delta p_{y}^{ \pm}=-\frac{e}{E_{0}^{ \pm}} \int_{\text {slice }} E_{y}^{\mp} d s
\end{aligned}
$$

where $E_{x}$ and $E_{y}$ are the transverse electric fields and $E_{0}$ is the energy of the synchronous particle. Here we have assumed that the particles are ultrarelativistic and $E_{0}=$ $c p_{0}$.

After the beam-beam (BB) kick, we reversed the operations to move the particles back to the interaction point.

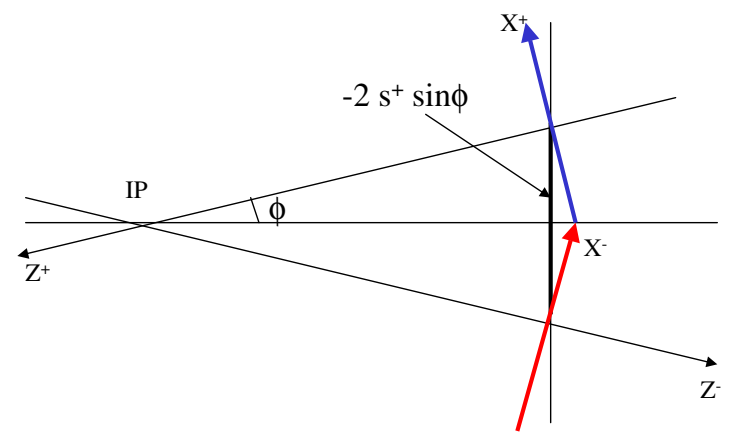

FIG. 8. (Color) Two colliding slices at their actual collision point with an angle $2 \times \phi$. The colors also indicate the coordinates at which the transverse beam-beam forces are calculated.
The whole process can be summarized as

$$
\mathcal{T}^{ \pm}\left(s^{ \pm}, \phi\right) \cdot \mathcal{O}_{\mathrm{BB}}^{\mp}\left(x^{ \pm}, y^{ \pm}\right) \cdot \mathcal{T}^{ \pm}\left(s^{ \pm}, \phi\right)^{-1},
$$

where

$$
\mathcal{T}^{ \pm}\left(s^{ \pm}, \phi\right)=\mathcal{D}_{z}^{ \pm}\left(s^{ \pm}\right) \cdot \mathcal{R}_{y}^{ \pm}(\phi) \cdot \mathcal{S}_{x}^{ \pm}\left(s^{ \pm}, \phi\right) \cdot \mathcal{R}_{y}^{ \pm}(\phi),
$$

and $\mathcal{O}_{\mathrm{BB}}^{\mp}\left(x^{ \pm}, y^{ \pm}\right)$represents the operator for the beambeam kick in Eqs. (20) and (21). Here we use the map convention. Namely, the operator on the left acts on a function of the canonical coordinates first and the dot represents the concatenation of the two maps.

The illustration of two colliding slices is a little oversimplified because a particle is likely not to be on any of the slices. For any given particle, we use a linear interpolation of the forces between two adjacent slices. Please also note that $\phi=-11 \mathrm{mrad}$ to match the KEKB configuration and coordinate conventions if the superscript \pm is used to denote positron and electron beams, respectively.

\section{CRAB CAVITY}

The main effect of a horizontal crab cavity is to tilt the beam in the $x-z$ plane. It can be described by a Hamiltonian

$$
H_{\text {crab }}=q_{c} x \sin \left(k_{\mathrm{rf}} l\right),
$$

where $q_{c}=V_{c} / E_{0}, k_{\mathrm{rf}}=2 \pi f_{\mathrm{rf}} / c$, and $V_{c}$ is the voltage of crab cavity. The kick to a particle is given by

$$
\Delta p_{x}=-q_{c} \sin \left(k_{\mathrm{rf}} l\right), \quad \Delta \delta=q_{c} k_{\mathrm{rf}} x \cos \left(k_{\mathrm{rf}} l\right) .
$$

Since a single crab cavity is installed in each ring in $\mathrm{KEKB}$, it mainly generates a wave of "crab dispersion"

$$
\zeta_{x}=-\frac{q_{c} k_{\mathrm{rf}} \sqrt{\beta_{c} \beta_{x}} \cos \left(\Psi_{c}-\mu_{x} / 2\right)}{2 \sin \left(\mu_{x} / 2\right)},
$$

where $\Psi_{c}$ is the difference of betatron phase between the observation point and the crab cavity and $\mu_{x} / 2 \pi$ is the horizontal tune. This formula is used to set the crab voltage in the simulation to compensate the crossing angle at the interaction point. Both crab cavities, though in different rings, are located in the Nikko site in our simulations. The actual circulating direction of beams is also implemented in the simulation.

TABLE II. Parameters associated with crab cavities in simulation. The $\mathrm{rf}$ frequency $f_{\mathrm{rf}}=509 \mathrm{MHz}$.

\begin{tabular}{lccc}
\hline \hline Parameter & Description & $e^{+}$ & $e^{-}$ \\
\hline$V_{c}(\mathrm{MV})$ & Crab voltage & -0.8755 & -1.618 \\
$\beta_{c}(\mathrm{~m})$ & Beta $x$ at crab cavity & 85 & 130 \\
$\Psi_{c}$ & phase to IP & $\pi / 2$ & $\pi / 2$ \\
\hline \hline
\end{tabular}


The main parameters for crab cavities are tabulated in Table II. We have used other parameters such as vertical beta functions at the cavity in our simulation. Their values are set according to the design. Since they do not affect the results of the simulation, we do not list them.

\section{BEAM-BEAM SIMULATION}

To implement the longitudinal wakefield into the beambeam simulation, one needs to change the tracking process in the arc. To keep things simple, we retain everything about the transverse dimensions and only modify the longitudinal dynamics according to the algorithms outlined in the subsection of "simulation with macroparticles." One has to keep in mind that $\beta_{\delta}=\sigma_{\delta} / \sigma_{z}, \nu_{s}$ is negative, and $l=-z$ in our canonical coordinates and transform the coordinates between $\delta, l$ and $q=-l / \sigma_{z}, p=-\delta / \sigma_{\delta}$. For every turn, we use 22 steps of $\theta$ to keep a thousand steps in one period. Finally, since the longitudinal distribution is not a Gaussian anymore, we adjust the rf phase to align longitudinally the beams according to their centers calculated with the Haissinski distributions. Also, the longitudinal positions of the colliding slices are adjusted according to the average value of the rms for the two Haissinski distributions at the beginning of the simulation. Usually, we initialize a Haissinski distribution of the macroparticles to start.

In addition to the impedance models and crab parameters, we have a list of main parameters of KEKB in Table III used in our simulation. One major difference from our previous simulation without longitudinal wake is that the bunch length is dynamically determined in the equilibrium. That is why we list only the zero-current bunch length in the table as an input. Since we always vary the currents with a fixed ratio, the bunch populations in the table merely define a reference case. For the results shown later, they are the second highest points in beam

TABLE III. The main parameters of KEKB. The ring circumferences $C=3016$ meter and the crossing angle is $2 \times 11 \mathrm{mrad}$.

\begin{tabular}{lccc}
\hline \hline Parameter & Description & $e^{+}$ & $e^{-}$ \\
\hline$E(\mathrm{GeV})$ & Beam energy & 3.5 & 8.0 \\
$N\left(10^{10}\right)$ & Bunch population & 8.66 & 5.05 \\
$\beta_{x}^{*}(\mathrm{~cm})$ & Beta $x$ at the IP & 80.0 & 80.0 \\
$\beta_{y}^{*}(\mathrm{~mm})$ & Beta $y$ at the IP & 5.9 & 5.9 \\
$\epsilon_{x}(\mathrm{~nm}-\mathrm{rad})$ & Emittance $x$ & 18.0 & 24.0 \\
$\epsilon_{y}(\mathrm{~nm}-\mathrm{rad})$ & Emittance $y$ & 0.18 & 0.24 \\
$\nu_{x}$ & $x$ tune & 45.508 & 45.515 \\
$\nu_{y}$ & $y$ tune & 43.5801 & 41.5801 \\
$\nu_{s}$ & $z$ tune & 0.024 & 0.022 \\
$\sigma_{z}(\mathrm{~mm})$ & Bunch length $\left(I_{b}=0\right)$ & 4.58 & 5.22 \\
$\sigma_{\delta}\left(10^{-4}\right)$ & Energy spread $\left(I_{b}=0\right)$ & 7.27 & 6.68 \\
$\tau_{t}(\mathrm{turn})$ & $x, y$ damping time & 4000 & 4000 \\
$\tau_{s}($ turn $)$ & $z$ damping time & 2000 & 2000 \\
\hline \hline
\end{tabular}

currents. The present operating currents are near to the third highest points, which are $20 \%$ lower.

\section{A. Specific luminosity}

For every simulation, we track 14000 particles for each beam in 10000 turns to obtain equilibrium distributions of two colliding beams in the six-dimensional phase space. The luminosity is numerically calculated with the overlapping integral. Both crab cavities on and off are simulated with various beam currents using the same parameters and code. The results are shown in Fig. 9 in specific luminosity compared with the measurements. Clearly, the results reconfirm the previous prediction that the luminosity should be doubled with the crab crossing. But our simulations do not explain why there is a significant drop of luminosity at higher beam currents. Our results imply that there is still room for improvement of KEKB. Most likely, the extra degradation in the measurements is due to the machine errors such as coupling, dispersions, and chromaticity which are not included our simulations. Since there are too many possibilities for the imperfections, it is beyond our investigation.

By including the longitudinal wake, our simulations become truly three dimensional. As shown in Fig. 10, the bunch indeed lengthens as the beam intensity increases. As a result, the horizontal beam sizes increase accordingly because of the crabbing at the interaction point. At the operating current, the horizontal beam sizes of the two beams are well matched at 130 microns. In the vertical plane, both beams are equally around 1 micron in size but increase significantly above the operating currents, generating long tails as shown in Fig. 11. The long tails could cause poor beam-beam lifetimes.

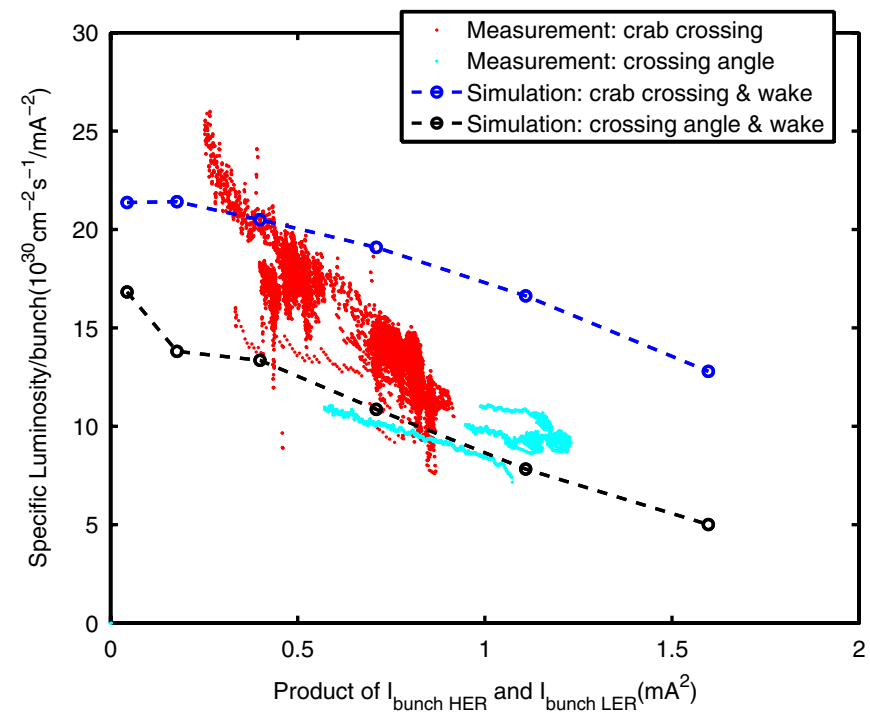

FIG. 9. (Color) Comparison of measured and simulated specific luminosity as a function of the product of bunch currents with/ without crab cavities. 

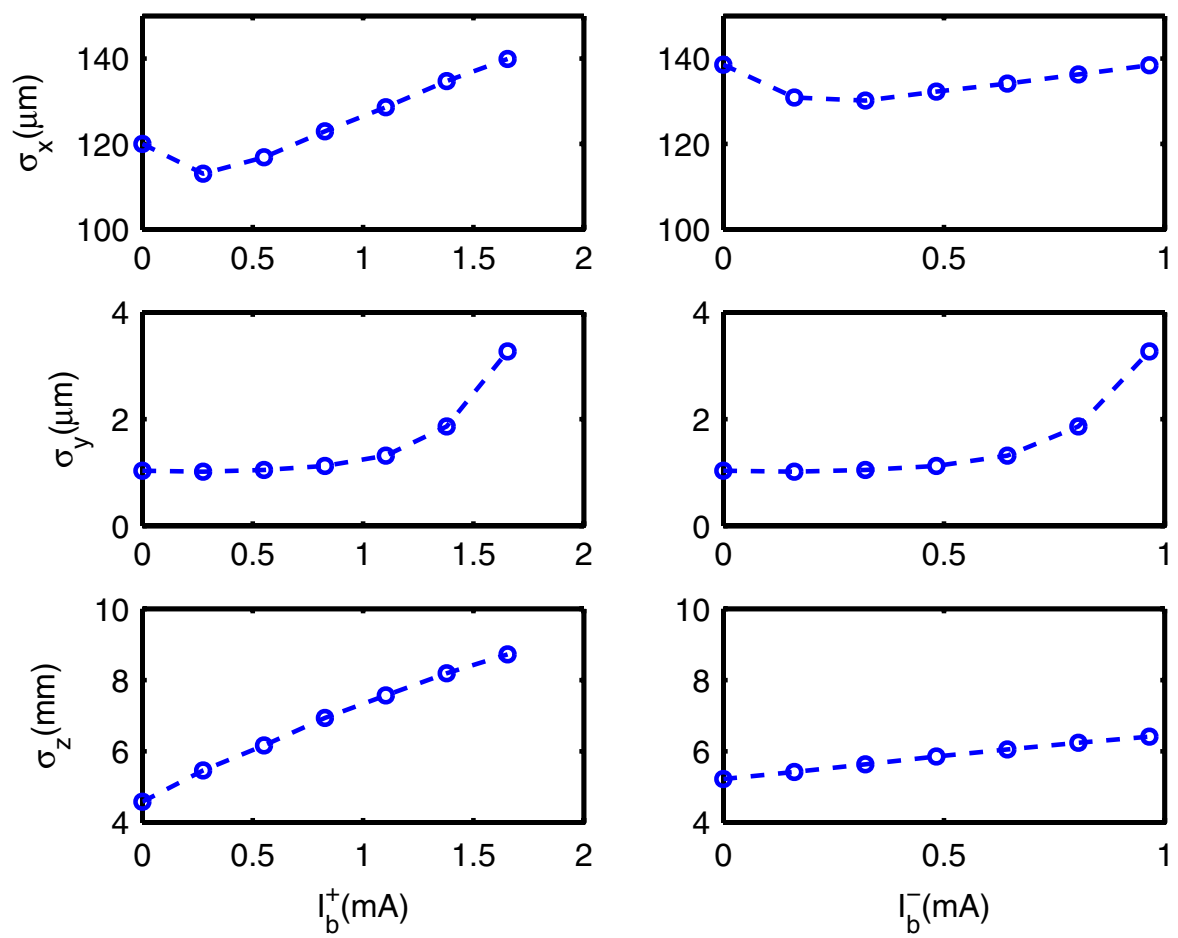

FIG. 10. (Color) Beam sizes in all three dimensions of colliding beams as a function of its current increased with a fixed ratio to the other.

Another feature of the longitudinal dynamics is the potential-well distortion described by the Haissinski distribution. Indeed, the longitudinal distribution of the elec- tron beam in its equilibrium as shown in Fig. 11 is asymmetric and matches its Haissinski distribution. This is a confirmation that the longitudinal wake has been
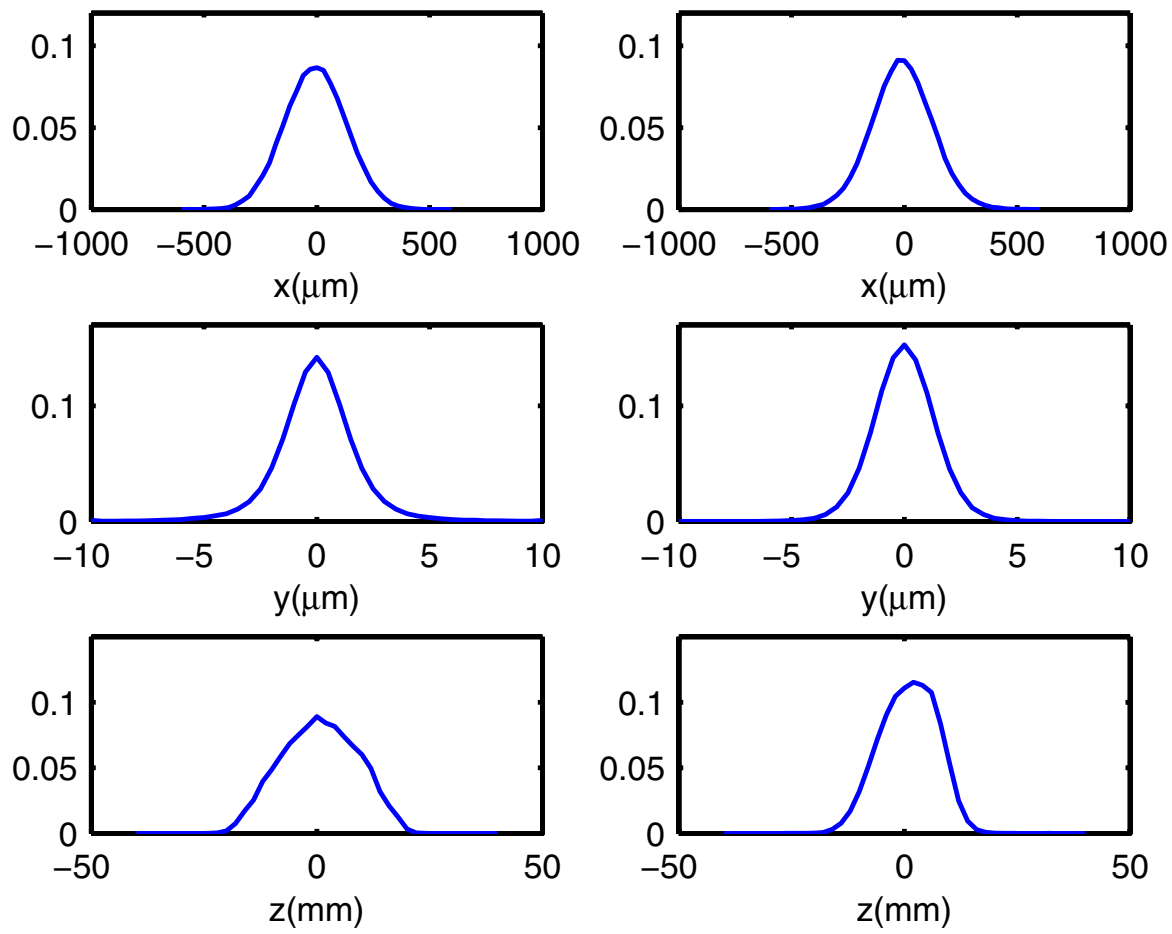

FIG. 11. (Color) Equilibrium distributions of colliding bunches at $1.38 \mathrm{~mA} / 0.80 \mathrm{~mA}$. Left columns are positron bunch and right for electron bunch. 

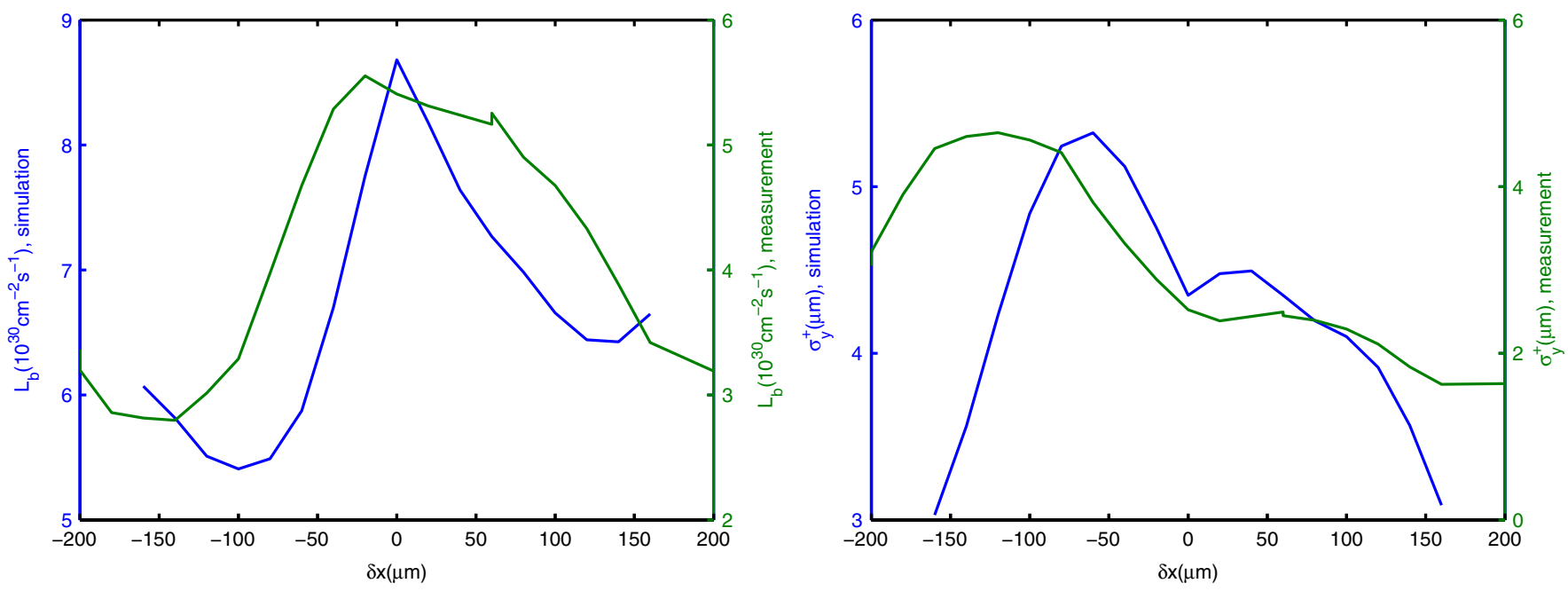

FIG. 12. (Color) Comparison of the simulation with a typical measurement at high beam currents. The figure on the left is the bunch luminosity and the right the vertical size of the positron beam at the IP.

implemented in the beam-beam program correctly since we know the threshold of microwave instability in HER is well above $1.0 \mathrm{~mA}$. On the other hand, the distribution of the positron beam at $1.38 \mathrm{~mA}$ deviates from its initial Haissinski solution, which is also expected because the current is well above the threshold as we discussed in the previous section.

\section{B. Horizontal scan}

It was a well-known mystery [20] that there was an asymmetry when the horizontal beam-beam scan was performed. The asymmetry became somewhat smaller after the crab cavities were installed in the rings. To understand this, we made simulations with horizontal offsets of the electron beam. When the crab cavities were turned off, we saw an asymmetry in luminosity and the vertical beam size of the positron beam as shown in Fig. 12, while the vertical size of the electron barely changed. The measurement was carried out in the year 2004 when the KEKB was operated without the crab cavities. Aside from a few differences of the lattices, the bunch currents in the measurement were $0.93 \mathrm{~mA} / 0.73 \mathrm{~mA}$, compared to the values $1.38 \mathrm{~mA} / 0.80 \mathrm{~mA}$ in the simulation. As a result, some deviations are expected. Because of the differences in the bunch currents, we adjusted the scales in the figure for a better comparison.

When the crab cavities are on, the asymmetry is significantly reduced to a negligible level. Clearly, the asymmetry originated from the difference of longitudinal distributions between positron and electron beams. As shown in Fig. 11, the crossing angle collision merely projected it into the horizontal plane. Since the crab crossing makes an effective head-on collision, the asymmetry disappears. We believe that on and off observations of the asymmetry are a reflection of machine imperfections.

\section{CONCLUSION}

Our study of the microwave instability was remarkably successful. We have shown that the simple broadband impedance models enabled us to explain many measurements and observations including the bunch shortening and lengthening, the shapes of beam profiles at various beam currents, and the thresholds of microwave instability and the bursting modes. Most important, its prediction of the growth of the energy spread was confirmed by the measurement using the particle detector Belle. The success can be attributed to several progresses we have made. First, we developed a PIC code and demonstrated that it can achieve the same accuracy provided by the VFP solver. Second, we found that it is critical to use the results of the simulation to fit the measured bunch length because the microwave instability contributes additional lengthening to the Haissinski solutions. Finally, we learned that shapes of the distributions were essential to narrow down the type of impedances.

Using a completely different method of simulation including the longitudinal wakefield, we reconfirm the main results by Ohmi who predicted that crab cavities would lead to an increase of luminosity by a factor of 2 . However, the luminosity of our simulation should be considered as an upper bound of the achievable value since the machine errors are not included in the simulation. The fact that the actual luminosity is significantly less than the predicted value shows a lack of understanding of the beam-beam limit. Our study indicates that the beam-beam limit may well be determined by the machine imperfections, most likely from the linear optics and stability of the storage rings.

Longitudinal impedances are successfully implemented and integrated into the beam-beam simulation. The simulation becomes truly three dimensional. The potential-well 
distortion and bunch lengthening are included. When the crossing angle is not compensated, we have shown that the asymmetry in the horizontal beam-beam scan is a result of the asymmetry in the longitudinal distributions.

In general, one should not simply fit the measured bunch profiles to Haissinski distributions because the microwave instability could enlarge the bunch further at higher currents as we have seen in the LER. We believe that at the operating current of $1.0 \mathrm{~mA}$, the positron beam at KEKB is above the threshold of microwave instability. As a result, its energy spread increases about $20 \%$, which may enhance the efforts of synch-betatron resonances.

Although the microwave instability does not cause any intrinsic emittance growth in the transverse dimensions, it does lead to larger effective beam sizes when dispersion and crab dispersion are present. This effect could lead to larger vertical beam sizes at the interaction point and therefore degrade the luminosity.

\section{ACKNOWLEDGMENTS}

Y. Cai would like to thank Y. Funakoshi for his invitation to visit KEK, where the work was done. In particular, his special thanks goes to all members in the KEKB term for their help and hospitality during his visit. We greatly appreciate Bob Warnock for providing his numerical solvers for the Haissinski's and the VFP equations. This work was supported by the U.S. Department of Energy, under Contract No. DE-AC02-76SF00515.

[1] G. Vignola et al., 1996 European Particle Accelerator Conference (Institute of Physics Publishing, Sitges, Spain, 1996), p. 22.
[2] C. Zhang et al., Reports No. IHEP-AC-Report/2002-01 and No. BEPCII-Report/2002-01, 2002.

[3] F. Ruggiero and F. Zimmermann, Phys. Rev. ST Accel. Beams 5, 061001 (2002).

[4] M. Bona et al., Reports No. SLAC-R-856, No. INFN-AE07-02, and No. LAL-07-15, 2007.

[5] K. Oide et al., Report No. KEK-Report-95-7, 1995.

[6] K. Ohmi et al., Phys. Rev. ST Accel. Beams 7, 104401 (2004).

[7] Y. Funakoshi et al., Phys. Rev. ST Accel. Beams 10, 101001 (2007).

[8] M. Sands, Report No. SLAC-Report-121, 1979.

[9] Alexander W. Chao, Physics of Collective Beam Instabilities in High Energy Accelerators (Wiley, New York, 1993).

[10] K. Oide and K. Yokoya, Report No. KEK-Preprint-90-10, 1990.

[11] R. Warnock and J. Ellison, Report No. SLAC-PUB-8404, 2000.

[12] J. Haissinski, Nuovo Cimento Soc. Ital. Fis. B 18, 72 (1973).

[13] S. Heifets, Report No. SLAC-PUB-12122, 2006.

[14] T. Ieiri and H. Koiso, in Proceedings of the 14th Symposium on Accelerator Science and Technology, KEK, Tsukuba (Particle Accelerator Society of Japan, Tsukuba, Japan, 2003), p. 443.

[15] S. Heifets et al., Report No. SLAC-PUB-6989, 1996.

[16] K. Hirata, Phys. Rev. Lett. 74, 2228 (1995).

[17] K. Ohmi, Phys. Rev. E 62, 7287 (2000).

[18] E. Forest, M. F. Reusch, D. L. Bruhwiler, and A. Amiry, Part. Accel. 45, 65 (1994).

[19] Y. Cai, A. W. Chao, S. I. Tzenov, and T. Tajima, Phys. Rev. ST Accel. Beams 4, 011001 (2001).

[20] Y. Funakoshi, "Beam-beam effects observed at the KEKB," Factories2001 workshop held at Cornell University.

[21] K. Bane and C. Ng, Report No. SLAC-PUB-6254, 1993. 